\section{Perfil de utilização de medicamentos antineoplásicos entre indígenas atendidos pelo Sistema Único de Saúde}

\author{
Profile of antineoplastic medication among \\ indigenous people treated in the Brazilian Unified \\ National Health System
}

\section{Perfil de utilización de medicamentos antineoplásicos entre indígenas atendidos por el Sistema Único de Salud brasileño}

COMUNICAÇÃO BREVE

BRIEF COMMUNICATION

\author{
Camila Alves Matos 1,2 \\ Claudia Garcia Serpa Osorio-de-Castro 3 \\ Carlos E. A. Coimbra Jr. 3 \\ Mario Jorge Sobreira da Silva 1
}

\section{Resumo}

Estima-se que no Brasil existam cerca de 900 mil indígenas (0,4\% da população do país) pertencentes a mais de 300 etnias diferentes. Porém, informações sobre a epidemiologia do câncer e sobre os medicamentos antineoplásicos utilizados no tratamento desses indivíduos são escassas. Os objetivos do presente estudo são identificar os medicamentos antineoplásicos utilizados e descrever o perfil de utilização deles na população indígena tratada no Brasil, além de descrever o perfil epidemiológico dos usuários. Foi realizado um estudo do tipo ecológico que envolveu os atendimentos ambulatoriais de quimioterapia, realizados no período de janeiro de 2014 e dezembro de 2018, em todo o Brasil. A fonte de coleta dos dados utilizada foi o Sistema de Informação Ambulatorial, do Departamento de Informática do SUS, considerando apenas as Autorizações de Procedimentos de Alta Complexidade para Quimioterapia. Foram identificadas 2.425 autorizações para procedimentos de quimioterapia destinadas ao tratamento de pacientes indígenas. Um total de 2.292 autorizações, para um total de 210 indivíduos, foram analisadas. Observou-se um aumento do número de autorizações e de indígenas em uso de quimioterapia ao longo do periodo, maior concentração de indivíduos residentes e em tratamento na Região Sul do país, diversidade de tipos de tumores diagnosticados $(n=63) e$ de esquemas terapêuticos utilizados durante o tratamento $(n=107)$. O presente estudo trouxe um novo panorama sobre o perfil epidemiológico dos usuários indígenas sob tratamento quimioterápico no Sistema Único de Saúde. Os achados contribuem para o preenchimento da atual lacuna de conhecimento a respeito da saúde indígena no Brasil.

Saúde de Populações Indígenas; Antineoplásicos, Sistema Único de Saúde

\author{
Correspondência \\ C. A. Matos \\ Instituto Nacional de Câncer José Alencar Gomes da Silva. \\ Rua Marquês de Pombal 125, 3o andar, Rio de Janeiro, RJ \\ 20230-240, Brasil. \\ camilamatos@id.uff.br \\ 1 Instituto Nacional de Câncer José Alencar Gomes da Silva, \\ Rio de Janeiro, Brasil. \\ 2 Universidade Federal Fluminense, Niterói, Brasil. \\ 3 Escola Nacional de Saúde Pública Sergio Arouca, \\ Fundação Oswaldo Cruz, Rio de Janeiro, Brasil.
}




\section{Introdução}

Segundo estimativas recentes para o triênio 2020-2022, a incidência de câncer no Brasil é de aproximadamente 625 mil novos casos por ano 1 . Embora o diagnóstico precoce e tratamento adequado contribuam para o bom prognóstico, as estratégias para o controle do câncer enfrentam problemas na organização de serviços de saúde e no desenvolvimento de ações 2 , promovendo desigualdades regionais e étnico-raciais de acesso que influenciam seu desfecho. Essas disparidades podem ser agravadas quando se consideram populações específicas, como os indígenas 3 .

Estima-se que no Brasil existam cerca de 900 mil indígenas (0,4\% da população do país) pertencentes, aproximadamente, a 300 etnias diferentes 4 . Diferenças nos padrões de ocorrência de neoplasias e cobertura de serviços de saúde em oncologia destinados ao atendimento da população indígena vêm sendo demonstradas ${ }^{3}$. Não obstante, são escassas as informações sobre tratamentos antineoplásicos utilizados nessa população.

Este estudo objetivou identificar os medicamentos antineoplásicos e descrever o perfil de utilização entre os usuários indígenas submetidos à quimioterapia, no período de 2014 a 2018.

\section{Método}

Trata-se de estudo do tipo ecológico que envolveu os atendimentos ambulatoriais de quimioterapia realizados no período de janeiro de 2014 a dezembro de 2018, em todo o Brasil, em unidades do Sistema Único de Saúde (SUS) habilitadas para tratamento oncológico. Foram investigados pacientes indígenas que apresentavam o câncer como diagnóstico principal, de acordo com a $10^{a}$ revisão da Classificação Internacional de Doenças (CID-10).

A fonte de dados foi o Sistema de Informação Ambulatorial (SIA-SUS), do Departamento de Informática do SUS (DATASUS), considerando apenas as Autorizações de Procedimentos de Alta Complexidade para Quimioterapia (APAC-Quimio). O número de procedimentos autorizados não corresponde necessariamente ao número de pacientes atendidos nas unidades habilitadas, pois cada autorização é válida para até três meses 5 .

A busca dos dados foi realizada entre abril e julho de 2019. Os pacientes foram identificados pela informação disponível no campo Raça/Cor (código 5). Foram consideradas as autorizações categorizadas como iniciais (código 1). Excluíram-se as APAC-Quimio destinadas ao tratamento de enfermidades com CID-10 D37-D48, que correspondem às neoplasias de comportamento incerto ou desconhecida, e aquelas que apresentavam sinalização de tratamento anterior. Para evitar duplicidades de identificação de sujeitos, foi utilizada a primeira autorização que apresentava o mesmo código do Cartão Nacional de Saúde.

Para caracterização dos tratamentos, foram utilizadas todas as autorizações constantes da base de dados. Coletaram-se as variáveis: sexo, idade, local de residência (município e Unidade da Federação - UF), local de tratamento (município e UF), CID-10 (principal, secundário e por topografia), presença de linfonodos regionais invadidos, estadiamento, grau histológico e esquema de quimioterapia registrado. Os dados foram tabulados e analisados no aplicativo Microsoft Excel (https://products.office. com/). A idade foi categorizada em: $0-18$ anos (pediatria) e $>18$ anos (adultos). Os dados de localização (residência e tratamento) foram agregados segundo as macrorregiões geográficas (Norte, Nordeste, Centro-oeste, Sul e Sudeste).

Calcularam-se as frequências absoluta e relativa (em percentual), e medidas de tendência central. As variáveis foram agrupadas tendo em conta dois eixos: caracterização socioeconômica e clínica dos pacientes e caracterização do tratamento utilizado.

\section{Resultados}

No período, foram identificadas 2.425 APAC-Quimio destinadas ao tratamento de indígenas com câncer no SUS. Excluíram-se 133 relacionadas ao tratamento dos CID-10 D37-D48. As 2.292 autorizações analisadas corresponderam a 210 indivíduos (Figura 1). 


\section{Figura 1}

Evolução história do número de Autorizações de Procedimentos de Alta Complexidade para Quimioterapia (APAC-Quimio) e o número de indígenas em uso de quimioterapia no Sistema Único de Saúde. Brasil, 2014-2018.

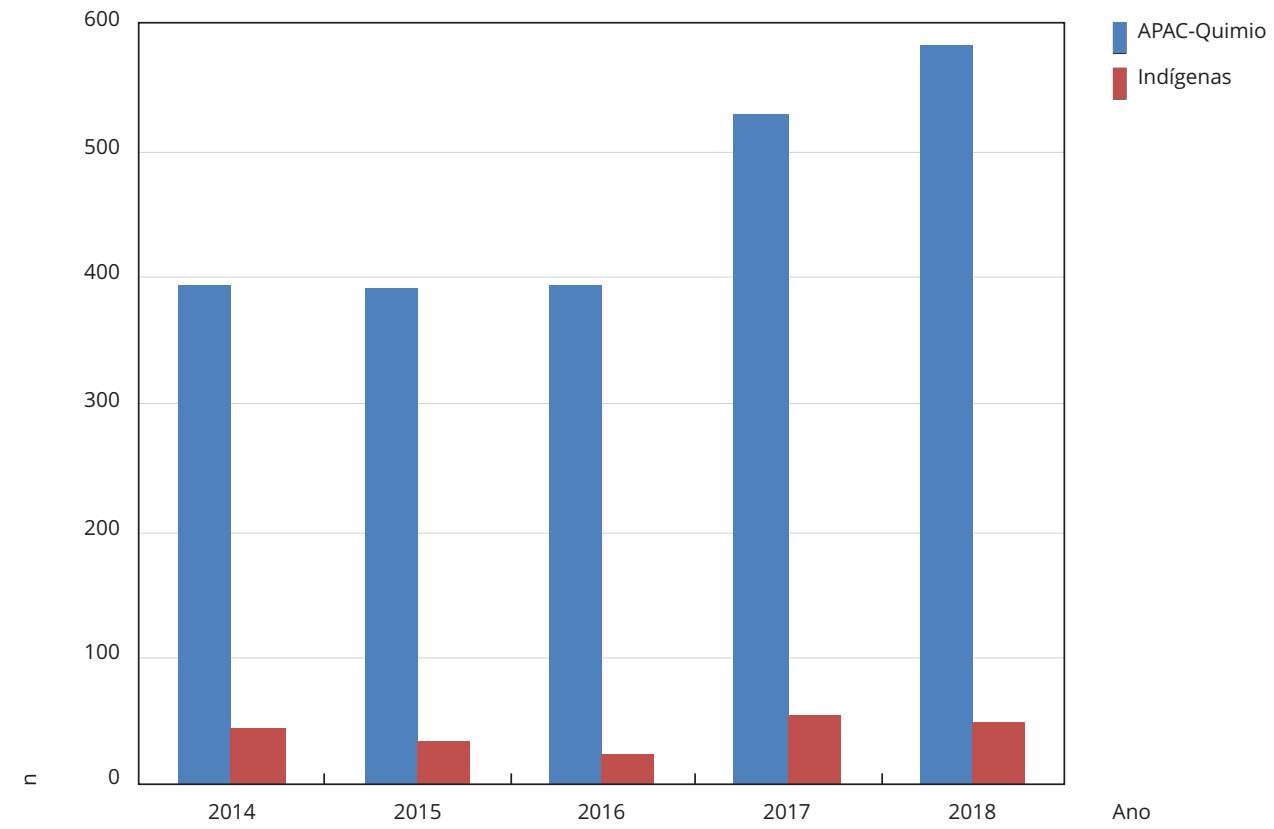

A maioria dos indígenas era do sexo feminino, com mediana de idade de 49 anos (0-95), residentes e tratados na macrorregião Sul do país. Os usuários residiam em 151 municípios e em 23 UF e foram tratados em 62 municípios e 23 UF.

Foram encontrados 63 diagnósticos diferentes, de acordo com a CID-10. Entre as mulheres, os tumores mais frequentes foram colo de útero $(32,8 \%)$, mama $(23,5 \%)$ e leucemia linfoblástica aguda $(4,2 \%)$. Entre os homens, as neoplasias mais recorrentes foram próstata $(29,7 \%)$, leucemia linfoblástica aguda (11\%) e leucemia mielóide crônica (6,6\%). Nos pacientes pediátricos, os cânceres mais tratados foram leucemia linfoblástica aguda (33,3\%), ossos (18,5\%), próstata $(7,4 \%)$, tronco cerebral $(7,4 \%)$ e doença de Hodgkin (7,4\%).

Cerca de 25\% dos pacientes apresentavam linfonodos regionais invadidos no início do tratamento e aproximadamente 55\% possuíam estadiamento III ou IV no momento do diagnóstico. As informações sobre grau histológico não estavam devidamente preenchidas, impedindo sua descrição e análise. A Tabela 1 apresenta as características sociodemográficas e clínicas da população estudada.

Verificaram-se 107 esquemas distintos de quimioterapia. Houve perda de informação em 1,1\% das APAC-Quimio. A Tabela 2 apresenta os esquemas de tratamento mais empregados, de acordo com os principais tipos de câncer identificados. 
Tabela 1

Dados sociodemográficos e clínicos da população indígena em uso de quimioterapia atendida pelo Sistema Único de Saúde. Brasil, 2014-2018.

\begin{tabular}{|c|c|c|}
\hline Características & n (APAC-Quimio inicial) & $\%$ \\
\hline \multicolumn{3}{|l|}{ Sexo } \\
\hline Feminino & 119 & 56,7 \\
\hline Masculino & 91 & 43,3 \\
\hline \multicolumn{3}{|l|}{ Faixa etária } \\
\hline Pediatria (0-18 anos) & 27 & 12,9 \\
\hline Feminino & 13 & 6,2 \\
\hline Masculino & 14 & 6,7 \\
\hline Adulto (> 18 anos) & 183 & 87,1 \\
\hline Feminino & 106 & 50,4 \\
\hline Masculino & 77 & 36,7 \\
\hline \multicolumn{3}{|l|}{ Região de residência } \\
\hline Norte & 50 & 23,8 \\
\hline Nordeste & 27 & 12,8 \\
\hline Sul & 62 & 29,5 \\
\hline Sudeste & 32 & 15,3 \\
\hline Centro-oeste & 39 & 18,6 \\
\hline \multicolumn{3}{|l|}{ Região de tratamento } \\
\hline Norte & 50 & 23,8 \\
\hline Nordeste & 27 & 12,8 \\
\hline Sul & 70 & 33,3 \\
\hline Sudeste & 31 & 14,8 \\
\hline Centro-oeste & 32 & 15,3 \\
\hline \multicolumn{3}{|l|}{ Clínica } \\
\hline Oncologia (tumores sólidos) & 164 & 78,1 \\
\hline Pediatria (0-18 anos) & 13 & 6,2 \\
\hline Adulto (> 18 anos) & 151 & 71,9 \\
\hline Hematologia & 46 & 21,9 \\
\hline Pediatria (0-18 anos) & 14 & 6,7 \\
\hline Adulto (> 18 anos) & 32 & 15,2 \\
\hline \multicolumn{3}{|l|}{ Linfonodos regionais invadidos } \\
\hline Sim & 53 & 25,2 \\
\hline Não & 72 & 34,3 \\
\hline Não avaliáveis & 85 & 40,5 \\
\hline \multicolumn{3}{|l|}{ Estadiamento } \\
\hline 0 & 6 & 2,9 \\
\hline 1 & 6 & 2,9 \\
\hline 2 & 37 & 17,6 \\
\hline 3 & 63 & 30,0 \\
\hline 4 & 53 & 25,2 \\
\hline Não especificado & 45 & 21,4 \\
\hline
\end{tabular}

APAC-Quimio: Autorizações de Procedimentos de Alta Complexidade para Quimioterapia. 
Tabela 2

Principais diagnósticos da população indígena em uso de quimioterapia no Sistema Único de Saúde e seus respectivos protocolos de tratamento. Brasil, 2014-2018.

\begin{tabular}{|c|c|c|}
\hline População/Tipo de câncer & Principais protocolos de tratamento utilizados & $\%$ \\
\hline \multicolumn{3}{|l|}{ Feminina } \\
\hline \multirow[t]{3}{*}{ Colo de útero } & Cisplatina & 42,0 \\
\hline & Paclitaxel + carboplatina & 18,5 \\
\hline & Cisplatina + 5-fluoruracila & 12,7 \\
\hline \multirow[t]{3}{*}{ Mama } & Tamoxifeno & 40,8 \\
\hline & Anastrozol & 21,5 \\
\hline & Fulvestranto & 14,5 \\
\hline \multirow[t]{3}{*}{ Leucemia linfoblástica aguda } & GBTLI * & 25,7 \\
\hline & Vincristina + asparaginase + daunorrubicina & 25,7 \\
\hline & St. Jude ** & 17,1 \\
\hline \multicolumn{3}{|l|}{ Masculina } \\
\hline \multirow[t]{3}{*}{ Próstata } & Acetato de leuprorrelina & 44,9 \\
\hline & Acetato de gosserrelina & 19,5 \\
\hline & Flutamida & 12,9 \\
\hline \multirow[t]{4}{*}{ Leucemia linfoblástica aguda } & GBTLI * & 31,9 \\
\hline & Vincristina + asparaginase + daunorrubicina & 23,1 \\
\hline & $\mathrm{BFM} * * *$ & 14,3 \\
\hline & St. Jude ** & 14,3 \\
\hline \multirow[t]{3}{*}{ Leucemia mielóide crônica } & Imatinibe & 86,2 \\
\hline & Dasatinibe & 9,6 \\
\hline & Nilotinibe & 2,4 \\
\hline \multicolumn{3}{|l|}{ Pediátricos } \\
\hline \multirow[t]{3}{*}{ Leucemia linfoblástica aguda } & GBTLI * & 33,8 \\
\hline & Vincristina + asparaginase + daunorrubicina & 29,3 \\
\hline & St. Jude ** & 13,5 \\
\hline \multirow[t]{3}{*}{ Ossos } & Metotrexato + cisplatina + doxorrubicina + ciclofosfamida & 51,8 \\
\hline & Carboplatina + etoposídeo + ifosfamida & 17,6 \\
\hline & Etoposídeo + ifosfamida & 7,0 \\
\hline \multirow[t]{2}{*}{ Próstata } & Acetato leuprorrelina & 94,9 \\
\hline & Acetato de gosserrelina & 5,1 \\
\hline Tronco-cerebral & Carboplatina + vincristina & 100,0 \\
\hline \multirow[t]{2}{*}{ Linfoma de Hodgkin } & $\begin{array}{l}\text { Rituximabe + doxorrubicina + bleomicina + } \\
\text { vimblastina + dacarbazina }\end{array}$ & 54,5 \\
\hline & $\begin{array}{c}\text { Ciclofosfamida }+ \text { vincristina }+ \text { procarbazina }+ \text { doxorrubicina }+ \\
\text { bleomicina }+ \text { vimblastina }+ \text { dacarbazina }\end{array}$ & 45,5 \\
\hline
\end{tabular}

* Grupo Brasileiro de Tratamento de Leucemia na Infância: metotrexato + citarabina + vincristina + daunorrubicina + L-asparaginase + ciclofosfamida + mercaptopurina + doxorrubicina + tioguanina;

** St. Jude Children's Research Hospital: etoposídeo + citarabina + vincristina + metotrexato + teniposídeo + mitoxantrona + peg-L-asparaginase + vimblastina + ciclofosfamida + mercaptopurina + fludarabina + idarrubicina + asparaginase;

*** Berlin-Frankfurt-Munster: vincristina + daunorrubicina + asparaginase + ciclofosfamida + citarabina + mercaptopurina + metotrexato. 


\section{Discussão}

Identificou-se aumento do número de autorizações e de indígenas em uso de quimioterapia ao longo do período. Em 11 de outubro de 2017, por meio da Portaria no 2663 do Ministério da Saúde 6, foi instituído um incentivo financeiro para a atenção especializada aos povos indígenas potencialmente influenciando o aumento observado. Entretanto, em comparação à população assistida pelo SUS, o número de indígenas em início de tratamento permaneceu baixo (DATASUS. http:// tabnet.datasus.gov.br/cgi/webtabx.exe?PAINEL_ONCO/PAINEL_ONCOLOGIABR.def, acessado em 12/Fev/2020), podendo estar relacionado à subnotificação da raça/cor indígena no preenchimento da APAC-Quimio 7 ou à dificuldade de acesso dessas populações ao tratamento do câncer 3 .

Quanto ao sexo dos pacientes tratados, indígenas e não indígenas se assemelham, provavelmente face ao diagnóstico resultante de políticas voltadas ao rastreamento do câncer ${ }^{1}$. Quanto à idade, destaca-se a frequência de $12,9 \%$ de quimioterapia no subgrupo pediátrico. Dados globais apontam que o câncer infanto-juvenil corresponde a aproximadamente $1 \%$ de todas as neoplasias diagnosticadas 8 , e que crianças indígenas apresentariam perfil semelhante ao de crianças não indígenas dentro de seu próprio país 9 .

As regiões Norte e Nordeste concentram o maior número de indivíduos indígenas em ambientes rurais e urbanos, respectivamente 10 , porém, a maioria dos indígenas residia e recebeu tratamento no Sul do Brasil. Dificuldades estruturais e de acesso aos serviços de oncologia 2, disparidades geográficas 5 e barreiras sócio-organizacionais 3 podem oferecer explicação para a questão, impondo aos pacientes indígenas a necessidade de realizar longas viagens para cidades distantes de suas residências em busca de diagnóstico e tratamento, nem sempre adequados.

$\mathrm{O}$ fato de o câncer de colo uterino ser o mais tratado entre as mulheres indígenas pode indicar parcialidade de rastreamento centrado em procedimentos efetivos de menor densidade tecnológica, como colpocitologia, em detrimento de outros, como mamografia; ou mesmo falta de cobertura vacinal para papilomavírus humano (HPV) 11. As leucemias estiveram entre os tumores mais tratados entre os homens. Contaminantes ambientais, campos eletromagnéticos e a infecção pelo vírus linfotrópico da célula humana I/II (HTLV) são fatores que podem contribuir para o desenvolvimento das leucemias entre povos indígenas 12 .

Neoplasias de próstata em pediatria são incomuns 8 , embora, entre adolescentes, estejam aumentando, em razão da obesidade, inatividade física, infecções sexualmente transmissíveis e exposição aos agentes cancerígenos 13 , o que pode justificar o resultado observado no presente estudo.

A presença de comprometimento linfonodal e a gravidade dos casos diagnosticados são preocupantes, podendo relacionar-se às deficiências da atenção primária em rastrear precocemente os casos de câncer entre indígenas, ou ao despreparo dos serviços de média complexidade em fornecer diagnósticos de maneira eficaz 2 .

Encontrou-se relação estreita entre os tratamentos fornecidos às populações indígenas e não indígenas para os mesmos cânceres, conforme os protocolos e diretrizes clínicas do Ministério da Saúde. Contudo, a menor taxa de captação de populações indígenas para atendimento oncológico promove piores resultados 3. É possível que, em parte, esse cenário seja causado pelos próprios serviços de saúde que não consideram as especificidades socioculturais dos povos indígenas, fundamental para o estabelecimento de vínculos de confiança entre usuários e profissionais de saúde 14.

A ausência e/ou inconsistência das informações disponibilizadas no SIA-SUS foi uma limitação do estudo. A incompletude de dados sobre o grau histopatológico dos tumores, por exemplo, contribui para a subestimação das taxas epidemiológicas do câncer na população indígena, e contribui para a desvalorização dos programas e políticas relacionados ao câncer em tais populações 15 . O perfil da população indígena tratada de câncer, portanto, é parcial, considerando que apenas a quimioterapia foi examinada. Não obstante, resultados inesperados foram obtidos e requerem investigação epidemiológica mais aprofundada, tais como a prevalência de câncer de próstata em população indígena infanto-juvenil. Os achados, todavia, contribuem para o preenchimento de lacunas de conhecimento sobre saúde indígena no Brasil, especialmente quanto ao acesso aos procedimentos de alta complexidade, retratados na assistência oncológica. Acredita-se que o estudo poderá contribuir para reflexão sobre o tema, incentivando o surgimento de novas questões e cooperando para a melhoria da atenção à saúde dos povos indígenas. 


\section{Colaboradores}

Todos os autores contribuíram em todas as etapas da produção do artigo.

\section{Informações adicionais}

ORCID: Camila Alves Matos (0000-0003-06474381); Claudia Garcia Serpa Osorio-de-Castro (0000-0003-4875-7216); Carlos E. A. Coimbra Jr. (0000-0003-4085-1080); Mario Jorge Sobreira da Silva (0000-0002-0477-8595).

\section{Referências}

1. Instituto Nacional de Câncer José Alencar Gomes da Silva. Estimativa 2020: incidência de câncer no Brasil. Rio de Janeiro: Instituto Nacional de Câncer José Alencar Gomes da Silva; 2019.

2. Silva MJS, O'Dwyer G, Osorio-de-Castro CGS. Cancer care in Brazil: structure and geographical distribution. BMC Cancer 2019; 19:987.

3. Aguiar-Junior PN, Stock GT, Lopes-Junior GL, Almeida MS, Tadokoro H, Gutierres BS, et al. Disparidades na epidemiologia e no tratamento de câncer nas populações indígenas brasileiras. Einstein (São Paulo) 2016; 14:330-7.

4. Santos RV, Teixeira P. O "indígena” que emerge do Censo Demográfico de 2010. Cad Saúde Pública 2011;27:1048-9.

5. Silva MJS, Melo ECP, Osorio-de-Castro CGS. Origin-destination flows in chemotherapy for breast cancer in Brazil: implications for pharmaceutical services. Ciênc Saúde Colet 2019; 24:1153-64.

6. Ministério da Saúde. Portaria no 2.663 , de 11 de outubro de 2017. Altera a Portaria de Consolidação no 6/GM/MS, de 28 de setembro de 2017, para redefinir os critérios para o repasse do Incentivo para a Atenção Especializada aos Povos Indígenas - IAEPI, no âmbito do Sistema Único de Saúde - SUS. Diário Oficial da União 2017; 16 out.

7. Nascimento ER, Wanderley AV, Chalu-Pacheco F, Almeida Júnior RC, Costa DF, Pereira GNL, et al. Perfil clínico e epidemiológico do câncer entre os índios do estado do Pará, Brasil. Rev Bras Oncol Clín 2015; 11:12-8.

8. Bhakta N, Force LM, Allemani C, Atun R, Bray F, Coleman MP, et al. Childhood cancer burden: a review of global estimates. Lancet Oncol 2019; 20:e42-53.

9. Valery PC, Moore SP, Meiklejohn J, Bray F. International variations in childhood cancer in indigenous populations: a systematic review. Lancet Oncol 2014; 15:e90-103.
10. Bastos JL, Santos RV, Cruz OG, Longo LAFB, Silva LO. Características sociodemográficas de indígenas nos censos brasileiros de 2000 e 2010: uma abordagem comparativa. Cad Saúde Pública 2017; 33 Suppl 1:e00085516.

11. Garnelo L, Horta BL, Escobar AL, Santos RV, Cardoso AM, Welch JR, et al. Avaliação da atenção pré-natal ofertada às mulheres indígenas no Brasil: achados do Primeiro Inquérito Nacional de Saúde e Nutrição dos Povos Indígenas. Cad Saúde Pública 2019; 35 Suppl 3:e00181318.

12. Borges MFSO, Koifman S, Koifman RJ, Silva IF. Mortalidade por câncer em populações indígenas no Estado do Acre, Brasil. Cad Saúde Pública 2019; 35:e00143818.

13. Bleyer A, Spreafico F, Barr R. Prostate cancer in young men: an emerging young adult and older adolescent challenge. Cancer 2020; 126:46-57.

14. Garnelo L. O SUS e a saúde indígena: matrizes políticas e institucionais do Subsistema de Saúde Indígena. In: Teixeira CC, Garnelo L, organizadores. Saúde indígena em perspectiva: explorando suas matrizes históricas e ideológicas. Rio de Janeiro: Editora Fiocruz; 2014. p. 107-42.

15. Sarfati D, Garvey G, Robson B, Moore S, Cunningham $R$, Withrow $D$, et al. Measuring cancer in indigenous populations. Ann Epidemiol $2018 ; 28: 335-42$. 
Abstract

Brazil has an estimated 900,000 indigenous people ( $0.4 \%$ of the country's population), belonging to more than 300 different ethnic groups. However, information is scarce on cancer epidemiology and the antineoplastic drugs used in treatment of these individuals. The study's objectives are to identify the antineoplastic drugs used and to describe the profile of use of these drugs in the indigenous population treated in Brazil, as well as to describe the patients' epidemiological profile. An ecological study was performed involving outpatient chemotherapy care from January 2014 to December 2018 throughout Brazil. The data source was the Outpatient Information System of the Brazilian Health Informatics Department, considering only Authorizations for High-Complexity Chemotherapy Procedures. We identified 2,425 authorizations for chemotherapy procedures for treatment of indigenous patients. Of these, 2,292 authorizations for a total of 210 individuals were analyzed. We found an increase in the number of authorizations and of indigenous persons receiving chemotherapy over the period, with a higher concentration of individuals living in and receiving treatment in the South of Brazil, plus a wide diversity in the types of diagnosed tumors $(n=63)$ and of treatment regimen used during the treatment $(n=107)$. The current study provided a new overview of the epidemiological profile of indigenous patients receiving chemotherapy in the Brazilian Unified National Health System. The findings help fill a current knowledge gap on indigenous people's health in Brazil.

Health of Indigenous Peoples; Antineoplastic Agents; Unified Health System

\section{Resumen}

Se estima que en Brasil existan cerca de 900 mil indígenas $(0,4 \%$ de la población del país) pertenecientes a más de 300 etnias diferentes. No obstante, la información sobre la epidemiología del cáncer y medicamentos antineoplásicos utilizados en el tratamiento de esos individuos es escasa. Los objetivos del presente estudio son identificar los medicamentos antineoplásicos utilizados, $y$ describir el perfil de utilización de esos medicamentos en la población indígena tratada en Brasil, así como describir el perfil epidemiológico de los usuarios. Se realizó un estudio de tipo ecológico, implicando la atención ambulatoria con quimioterapia, realizada en el período de enero de 2014 y diciembre de 2018 en todo Brasil. La fuente de recogida de datos utilizada fue el Sistema de Información Ambulatoria, del Departamento de Informática del SUS, considerando solamente las Autorizaciones de Procedimientos de Alta Complejidad para Quimioterapia. Se identificaron 2.425 autorizaciones para procedimientos de quimioterapia, destinadas al tratamiento de pacientes indígenas. Se analizaron 2.292 autorizaciones para un total de 210 individuos. Se observó un aumento del número de autorizaciones $y$ de indígenas en el tratamiento de quimioterapia a lo largo del período, con una mayor concentración de individuos residentes y en tratamiento en la región sur del país, con una diversidad de tipos de tumores diagnosticados $(n=$ 63) y de esquemas terapéuticos utilizados durante el tratamiento $(n=107)$. El presente estudio presentó un nuevo panorama sobre el perfil epidemiológico de los usuarios indígenas bajo tratamiento quimioterápico en el Sistema Único de Salud. Los hallazgos contribuyen a llenar la actual laguna de conocimiento con respecto a la salud indígena en Brasil.

Salud de Poblaciones Indígenas; Antineoplásicos; Sistema Único de Salud
Recebido em 28/Abr/2020

Versão final reapresentada em 30/Set/2020

Aprovado em 19/Out/2020 